\title{
Nekoze! - Monitoring and Detecting Head Posture while Working with Laptop and Mobile Phone
}

\author{
Katsuma Tanaka, Shoya Ishimaru, Koichi Kise \\ Osaka Prefecture University, Japan \\ \{katsuma, ishimaru\}@m.cs.osakafu-u.ac.jp \\ kise@cs.osakafu-u.ac.jp
}

\author{
Kai Kunze, Masahiko Inami \\ Graduate School of Media Design \\ Keio University, Yokohama, Japan \\ Email: \{kai,inami\}@kmd.keio.ac.jp
}

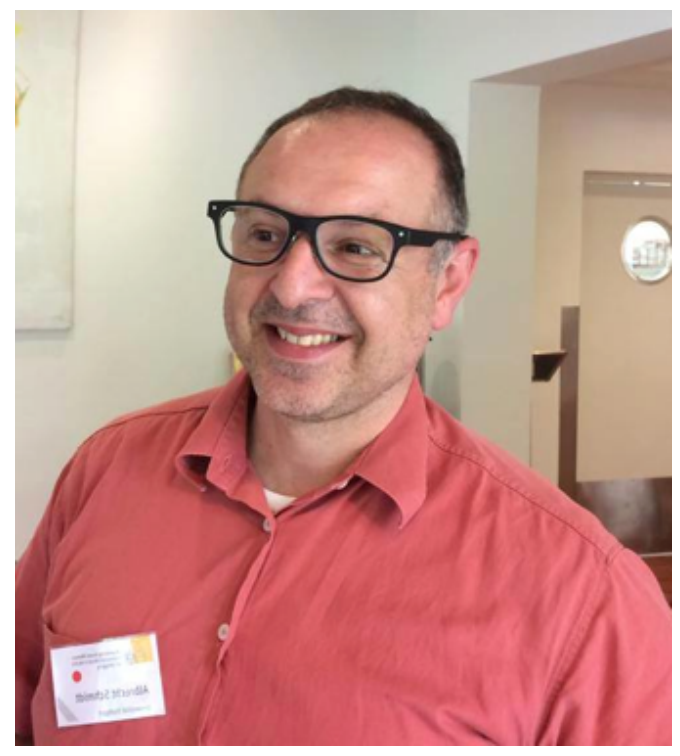

\begin{abstract}
Neck pain and other spine related injuries are on the rise. One potential cause is bad head posture while using digital devices. We present two systems to monitor and improve head posture for two of the common problematic cases: laptop and mobile phone use. The laptop system uses the front-facing camera. The mobile system the sensor data of a smart glasses prototype. Both systems work reasonable in an initial user study with 10 and 8 participants ( $72 \%$ detection on the laptop system, $100 \%$ for the mobile one). From the discussion, it seems users are especially interested in the smart glasses based monitoring implementation.
\end{abstract}

Keywords-eye wear computing; head posture; smart glasses

\section{INTRODUCTION}

As more and more people spend time on digital devices, injuries and chronic conditions related to this increased usage are also on the rise. Using computing devices with bad posture can lead to neck pain, spine problems, repetitive stress injuries, just to name a few.

However, so far most researchers focus on recommending regular breaks and to correct their posture over using altering their office (e.g. monitor adjustments, standing desks). They disregard the emerging ergonomic problems related to smart phones and tablets[5]. Especially, with increase of smartphone usage for longer periods of time, users tend to stay in unhealthy postures while writing text messages or playing mobile games. They bend their head forward too much. With the ease of access to smart phones, there is the concern of over-useage. This combined with the unhealthy head positions can lead to chronic neck pain, musculoskeletal disorders, repetitive stress injuries (RSI) or worse device-usage-related injuries[14].

We believe we need a new approach towards posture monitoring and improvement focusing on unobtrusive sensing and seamless interactions with the user to prevent bad posture and increase quality of life.

The contributions of this paper are as follows: (1) we describe an approach and a system to monitor head postures using a laptop and a mobile system using smart glasses. (2) We detect bad head posture (over an 19 degree sholder angle) for both systems. (3) We present a user study and expert evaluation for both system. Feedback indicates that our systems are already usable as is. The laptop system detected 72 of $100 \mathrm{bad}$ posture states. The mobile system detected all 100 during our initial user tests. (4) We discuss results and present recommendations for a head posture monitoring and improvement system.
Fig. 1. User wearing a J!NS MEME smart glasses prototype.

\section{RELATED WORK}

A couple of researchers work on dedicated Sensors. Denmans et al. use a system based on a bend sensor to detect bad head postures and give feedback to the users[4]. Sonne et al. embed sensors into a "Habit aware mouse"[12] focusing mostly on hand strain.

There is a lot of research focusing on detecting head postures using accelerometers [9], [1]. Yet, most focuses on augmented reality or other applications. The others, we see as complementary work as the hardware/algorithms are usually more complex using bigger dedicated sensing devices.

The focus of the research is also mostly on tracking rehabilitation progress instead of working on prevention. Arteaga et al. developed a system to help stroke patients from having bad posture[1]. They implemented a monitoring system placing accelerometers and actuators at different the body parts. They warn the patient over several modalities and also log bad postures for the doctor to check later. Yet, they did not focus on head posture. Along this lines, Daian et al. present an intelligent chair to measure posture in the office using force resistive sensors[3] focusing on body posture while sitting. 
There is also an Android app called "Text Neck Indicator"1. Yet it just monitors the angle of the smart phone not the head.

Close to our work is the research by Lee et al. and Shin et al. [10], [11]. Lee et al. present an algorithm to monitor the neck angle using a front facing camera on the smart phone. Yet, they don't present an evaluation for the system. Shin et al. use smartphone usage patterns (e.g. the ratio of SMSs to calls, the number of event-initiated sessions) to identify problematic usage patterns impacting social and heath aspects of users.

There are also a couple posture monitoring/improvement systems using computer vision. Jaimes et al. uses a camera to detect activities and related postures. They show a small icon on the computer screen to indicate the quality of posture related to the current activity[8], [7].

Posture monitoring systems have been proposed by Jaimes and Liu $[5,6]$. Their systems use computer vision to determine the user's activities through his/her posture. By creating postural awareness Jaimes helps the user to improve his/her posture by continually indicating posture quality via an icon [5].

Related to using smart glasses, Hong et al. use smart earphones with integrated accelerometer and gryroscope to monitor the posture [6]. Yet their goal is also not head angle determination. They monitor how long the user is not moving/staying in the same postures. The user can get a record of posture shifts during the day.

\section{APPROACH}

We decided to focus on head posture as it seems to be an increasing problem with the use of laptops and especially smart phones/tablets. The first question for us was what constitues a bad head position. Consulting ergonomics research, straining (or bad) head postures are defined in ergonomics over a head/shoulder angle higher than 19 degrees[2]. We focus on monitoring this head angle. Additionally, working with increases in neck angle (over 19 degrees) for over longer time are associated with loss in height, torque increases on the cervical spine, and increased discomfort[2], [13]. As seen from this work as well, most cases of bad posture happen during laptop use due to their bad ergonomic design and during mobile phone/tablet use. Therefore, we want to tackle theses cases and decided to explore both cases by designing initial systems for head posture detection.

Our goal is to do monitoring as unobtrusive as possible best with the devices themselves. That's why we decided first to use the front-facing camera on a laptop/tablet/phone only. Initial tests confirmed that on a laptop computer it's feasible. Without much trouble we could detect bad head postures using straight forward Open CV face detection libraries.

However, with early tests we had really bad results on the phones and tablets. Compared to the laptop for some users the face is not always full in the view of the camera. Detecting distances and posture is also more difficult due to the varying phone positions and angles to the face. Therefore we decided to include smart glasses. A lot of people already wear glasses for work on a computer or mobile screen. We did not want to

\footnotetext{
${ }^{1}$ https://play.google.com/store/apps/details?id=com.textneckbeta.gui\&hl=de
}

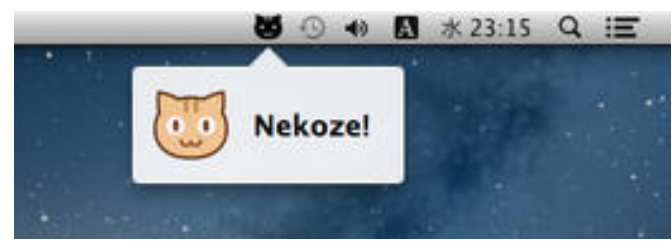

Fig. 2. Notification of the desktop Nekoze application

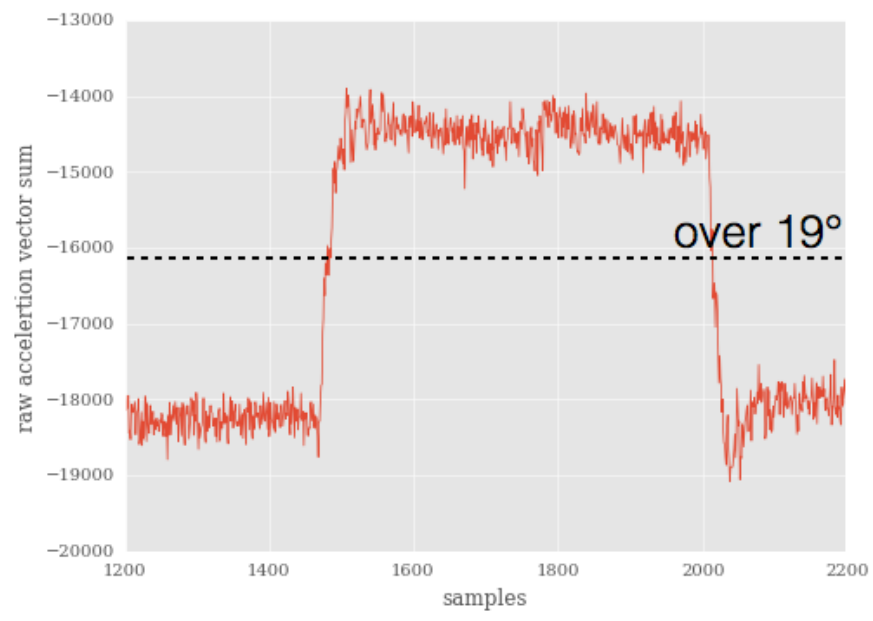

Fig. 3. The vector sum of the raw acceleration axis during a bad posture incident (head angle over 19 degrees). The change is easily visible only from the raw values.

use dedicated posture detection devices as some related work and head phones seemed to be too limited in use ( e.g. need to be taken out for social interactions). In the following we will discuss our first implementations of the two systems. It is to note that these are exploratory implementations to gather feedback.

\section{IMPLEMENTATIONS}

As mentioned, we focus on the laptop and mobile use cases. We implemented two Nekoze! prototypes one for each use case described in the following. Nekoze is Japanese meaning "to arch one's back like a cat". The word is used to refer to bad posture when working on a computer or mobile device.

\section{A. Nekoze! Laptop Prototype}

For the laptop system, we use an implementation that access the front-facing camera available on most systems. It applies face detection and detects if the face gets too close to the screen. The prototype is implemented Mac OS X.

As soon as the user gets too close to the screen, we have 3 stimuli: a notification on the top bar (see Fig 2), notification and sound, just sound, or a "screen flash" (screen brightness increases slightly and contrast is reduced ).

For the user study later, we had a calibration at the beginning of the session, with the user sitting straight.

\section{B. Nekoze! Mobile Prototype}

For the mobile prototype we use a J!NS MEME prototype and an iPhone. J!NS MEME are sensing glasses including 


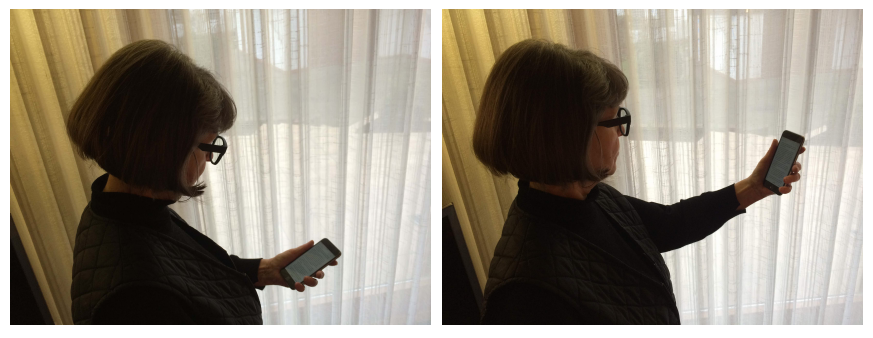

Fig. 4. A participant holding the phone in a straining posture with a head angle over 19 degrees (left) and in a neutral postion (right) under 19 degrees.

electrodes to detect eye movements and inertial motion sensors (accelerometer/gyroscope). For the system we just use the accelerometer. MEME streams the sensor data to a laptop or smartphone with a sampling rate of $60 \mathrm{~Hz}$ for the acceleration. Battery runtime for the current prototype is around 8 hours.

We use the raw accelerometer data from the glasses to estimate the head angle. Already simple thresholding performs quite well. Yet, we calculate the vector sum of $\mathrm{x}, \mathrm{y}, \mathrm{z}$ acceleration afterwards we apply a low pass filter (cutoff $1.5 \mathrm{~Hz}$ ) and use the acceleration vector sum value as input for a support vector regression to determine the head angle.

Fig 3 shows the raw accelerometer vector sum for a bad posture incident.

If the head angle is over 19 degrees, we use the following feedback mechanisms: Standard notification shown at the top of the mobile screen or a screen flash (changing brightness and contrast).

\section{INITIAL EVALUATION}

\section{A. User Studies}

Both studies were conducted in several offices and school environments indoors at desks where people tend to work with computers/laptops and for the mobile study standing on the floor in the kitchen, office or living rooms.

We had 10 participants (4 female, mean age 27, std 10) for the laptop study. We introduce the laptop system to the participants, show them the functionality and let them try it while playing a browser game and web surfing (between 5-15 min.). For the test we use notifications with sound. The users perform $10 \mathrm{x}$ straining posture (head angle over 19 degrees) and $10 \times$ normal posture positions in front of the system. After the test we interviewed them and presented them the alternative feedback mechanisms.

For the mobile study we had 8 participants ( 3 female, mean age 34 , std 8 ). We ask the users to put the smartphone into their pocket, take it out and use it as they regularly would. Measuring the head angle 5 of the 8 users showed a bad posture (head angle over 19 degrees).

After this test we introduce them to the background of our study and the mobile system. The users can try the system using a game (a puzzle game called 2045) or a longer text to read. We also record 10 x straining posture (head angle over 19 degrees) and $10 \mathrm{x}$ normal posture positions from the users (see Fig. 4).
Additionally, We ask 5 users to hold their head a specific angle $(5,15,20,25,30,35) 10$ times and record data. We determine the angle using a protractor. After applying the low pass filter and training the support vector regression we get an error of roughly 5 degrees with a standard deviation of 3 .

\section{B. Expert Evaluations}

For expert evaluation we discussed with two medical professional working in physical rehabilitation, specializing in neck and back related injuries.

After showing them the two systems, we conducted an informal interview and discussed with them. They liked the overall system designs and think it could already be used for patients. They prefer the mobile smart glasses solution as it is able to detect bad posture not only while sitting in front of a computer. However, for both systems they criticized the notifications. They informed us that chronic neck pain and similar symptoms are seldom from a couple of short bad posture incidents. What is potentially worse is one bad posture incident held for a long time (e.g. a user playing a game for 10 -20 minutes with bad head posture). Therefore, repeated notifications after 2-5 minutes of bad posture make more sense. Showing them the different feedback mechanisms both also preferred the screen flash. One professional mentioned that it might be too easy to ignore. She would introduce a more and more severe feedback (heavier flashing) the longer the posture was not changed. The other professional mentioned he would like to see a summary of a patients posture changes during day, to give better guidance and advice to his patients.

Regarding user interface and system, we interviewed to two HCI researchers. They also didn't like the notification type feedback too much, screen flash was also their preferred feedback. They also mentioned haptics (vibration) or an led in the glasses as other modalities to inform the user. One Expert remarked that it might be worth exploring if there are feedback possibilities so that the user does not actively need to change the position, but the system induces the change subconsciously (e.g. muscle stimulation).

\section{DISCUSSION}

For both user studies, users remarked they would like to use a system to track their posture during everyday life. 15 of the 18 users have trouble with the neck pain or a stiff neck sometimes.

Discussing the laptop system first, the notification or sound were not so attractive to 6 users, they preferred the screen flash. 2 users preferred the notifications without sound and 2 were indifferent. 7 users could imagine to use the system as is, for 2 any presented feedback was too annoying to use everyday. Yet, they could not give suggestions on what type of feedback they wanted instead. Our laptop system could detect 72 of the 100 bad posture states. For one user the system did not perform well as there were some lighting issues (sun backlight). 3 users voiced some frustration that the system was not working optimally (didn't detect their bad posture). One user mentioned he is using MacBreakZ ${ }^{2}$, a personal ergonomic assistant program designed to promote healthy and productive

\footnotetext{
${ }^{2}$ http://www.publicspace.net/MacBreakZ/
} 
computer use. It monitors the typing behavior and provides exercise breaks accordingly. He could imagine Nekoze! to do the same for back pain (not only for hands).

About the mobile system, all 8 users liked the idea of using smart glasses to detect. 5 users already wear everyday. Also here the users preferred the screen flash (all 8) compared to the notifications. Still 3 users found even the screen flash to irritating especially when reading. One user mentioned he would like to get just a vibration on the phone or better on the smart glasses. All straining posture states could be correctly classified by the system. One user asked if the glasses could also be used to monitor/analyze her steps, as she had trouble a couple of years ago related to uneven gait.

\section{CONCLUSION AND FUTURE WORK}

In this paper we presented 2 systems to detect straining had positions and designed initial user studies to collect feedback for further development as well as to evaluate the current systems performance. The results of our first posture monitoring and improvement systems seems encouraging. Especially smart glasses seem an interesting approach to track posture, as they can be used not only while working on the laptop but also during other everyday situations. e.g. reading a hardcover book.

Next we will start an exploratory user study to explore potential feedback ideas more. In term we will conduct also a larger experimental setup to validate the posture detection on the smart glasses. Several users and experts suggested to build out the long term monitoring for later review and custom interventions. This is also a direction we will explore more. Other important future work include improving the recognition system for the latop Nekoze! validating the detection for both systems on a larger population, implementing log/better feedback mechanisms and identifying how/when to intervene.

\section{ACKNOWLEDGMENT}

This work is partly supported by Kakenhi Grant-in-Aid B No. 26730095 and the CREST project from Japan Society for the Promotion of Science (JSPS).

\section{REFERENCES}

[1] Sonia Arteaga, Jessica Chevalier, Andrew Coile, Andrew William Hill, Serdar Sali, Sangheeta Sudhakhrisnan, and Sri H. Kurniawan. Low-cost accelerometry-based posture monitoring system for stroke survivors. In Proceedings of the 10th International ACM SIGACCESS Conference on Computers and Accessibility, Assets '08, pages 243-244, New York, NY, USA, 2008. ACM.

[2] RA Bonney and EN Corlett. Head posture and loading of the cervical spine. Applied Ergonomics, 33(5):415-417, 2002.

[3] Ioana Daian, AM Van Ruiten, Albertine Visser, and Senka Zubic. Sensitive chair: a force sensing chair with multimodal real-time feedback via agent. In Proceedings of the 14th European conference on Cognitive ergonomics: invent! explore!, pages 163-166. ACM, 2007.

[4] Carrie Demmans, Sriram Subramanian, and Jon Titus. Posture monitoring and improvement for laptop use. In $\mathrm{CHI}$ '07 Extended Abstracts on Human Factors in Computing Systems, CHI EA '07, pages 2357-2362, New York, NY, USA, 2007. ACM.

[5] KENNETH K HANSRAJ. Assessment of stresses in the cervical spine caused by posture and position of the head. Surgical technology international, 25:277-279, 2014.
[6] Dezhi Hong, Ben Zhang, Qiang Li, Shahriar Nirjon, Robert Dickerson, Guobin Shen, Xiaofan Jiang, and John Stankovic. Septimu: continuous in-situ human wellness monitoring and feedback using sensors embedded in earphones. In Proceedings of the 11th international conference on Information Processing in Sensor Networks, pages 159-160. ACM, 2012.

[7] Alejandro Jaimes. Sit straight (and tell me what i did today): a human posture alarm and activity summarization system. In Proceedings of the 2nd ACM workshop on Continuous archival and retrieval of personal experiences, pages 23-34. ACM, 2005.

[8] Alejandro Jaimes. Posture and activity silhouettes for self-reporting, interruption management, and attentive interfaces. In Proceedings of the 11th international conference on Intelligent user interfaces, pages 24-31. ACM, 2006.

[9] Soonmook Jeong, Taehoun Song, Hyungmin Kim, Miyoung Kang, Keyho Kwon, and Jae Wook Jeon. Human necks posture measurement using a 3-axis accelerometer sensor. In Computational Science and Its Applications-ICCSA 2011, pages 96-109. Springer, 2011.

[10] Hosub Lee, Young Sang Choi, and Sunjae Lee. Mobile posture monitoring system to prevent physical health risk of smartphone users. In Proceedings of the 2012 ACM Conference on Ubiquitous Computing, UbiComp '12, pages 592-593, New York, NY, USA, 2012. ACM.

[11] Choonsung Shin and Anind K. Dey. Automatically detecting problematic use of smartphones. In Proceedings of the 2013 ACM International Joint Conference on Pervasive and Ubiquitous Computing, UbiComp '13, pages 335-344, New York, NY, USA, 2013. ACM.

[12] Tobias Sonne and Kaj Grønbæk. Exploring new potentials in preventing unhealthy computer habits. In CHI' 13 Extended Abstracts on Human Factors in Computing Systems, CHI EA '13, pages 487-492, New York, NY, USA, 2013. ACM.

[13] Leon Straker, Kerry J Jones, and Jenni Miller. A comparison of the postures assumed when using laptop computers and desktop computers. Applied Ergonomics, 28(4):263-268, 1997.

[14] Jens Wahlström. Ergonomics, musculoskeletal disorders and computer work. Occupational Medicine, 55(3):168-176, 2005. 